\title{
Margination of White Blood Cells in Microcapillary Flow
}

\author{
Dmitry A. Fedosov, Julia Fornleitner, and Gerhard Gompper \\ Theoretical and Soft Matter Biophysics, Institute of Complex Systems and Institute for Advanced Simulation, \\ Forschungszentrum Jülich, 52425 Jülich, Germany
}

(Received 21 September 2011; published 11 January 2012)

\begin{abstract}
Margination of white blood cells (WBCs) towards vessel walls is an essential precondition for their efficient adhesion to the vascular endothelium. We perform numerical simulations with a two-dimensional blood flow model to investigate the dependence of WBC margination on hydrodynamic interactions of blood cells with the vessel walls, as well as on their collective behavior and deformability. We find WBC margination to be optimal in intermediate ranges of red blood cell (RBC) volume fractions and flow rates, while, beyond these ranges, it is substantially attenuated. RBC aggregation enhances WBC margination, while WBC deformability reduces it. These results are combined in state diagrams, which identify WBC margination for a wide range of flow and cell suspension conditions.
\end{abstract}

Introduction.-Leukocytes or white blood cells (WBCs) are part of our immune system, performing various organism defense functions. In order to reach the sites of action (e.g., inflammation), WBCs have to migrate to the vessel walls through a process called margination [1-3], adhere efficiently to vascular endothelium (mediated by adhesion proteins $[4,5]$ ), and further transmigrate into the surrounding tissues [6]. The efficiency of WBC adhesion in blood flow is correlated with the contact frequency of WBCs with the vessel walls, and thus with margination.

The mechanism for WBC margination in microvessels is related to the presence of red blood cells (RBCs) in blood. RBCs in microvessels migrate to the vessel center $[7,8]$. This process is governed by cell-wall hydrodynamic interactions [9] (often called lift force), which drive the cells away from the wall, and by cell-cell interactions, which tend to disperse RBCs [10]. WBC margination is believed to be a consequence of the competition between lift forces on RBCs and WBCs, where the lift force on a RBC is larger than that on a WBC due to the nonspherical discocyte shape and high deformability of RBCs. These interactions result in WBC margination (i.e., segregation), such that their concentration becomes higher near a vessel wall $[2,3]$.

Several blood and flow properties may contribute to WBC margination, which include hematocrit $H_{t}$ (i.e., RBC volume fraction), flow rate, vessel geometry, and RBC aggregability [1,3,11-14]. In vivo experiments on WBC margination in the mesenteric venules of rats [3] showed a substantial increase of the WBC adhesion rate (and consequently margination), with the decreasing flow rate at relatively high $H_{t}>0.45$. A different dependence of WBC margination on $H_{t}$ was reported in recent in vitro experiments on blood flow in microfluidic channels [14], where the strongest margination effect was found at intermediate $H_{t} \simeq 0.2-0.3$, while, at lower or higher $H_{t}$ values, WBC margination was attenuated. In contradiction to these experiments, recent results on WBC adhesion in glass capillaries [12] show no dependence of WBC adhesion, and therefore margination, on hematocrit. The effect of flow rate on WBC margination and adhesion seems to be consistent across the various studies [3,11,14] which showed a significant enhancement at low flow rates comparable with those in venular blood flow. In addition, RBC aggregation has been found to result in an increased WBC margination and adhesion $[3,11,12,14]$ at the vessel walls.

Simulations of a two-dimensional blood model in Ref. [13] showed increasing margination with decreasing flow rate, in agreement with experiments, but only a weak dependence on hematocrit. WBC margination is found to be insensitive to RBC deformability, which suggests that the $\mathrm{RBC}$ biconcave shape is the main governing parameter for the lift force on a RBC [13].

In order to integrate these various, sometimes apparently contradicting, observations into a coherent picture, a more extensive study of the dependence of WBC margination on hematocrit, flow rate, WBC deformability, and RBC aggregation is clearly necessary. We focus here on a twodimensional (2D) model of blood flow; see Fig. 1 and

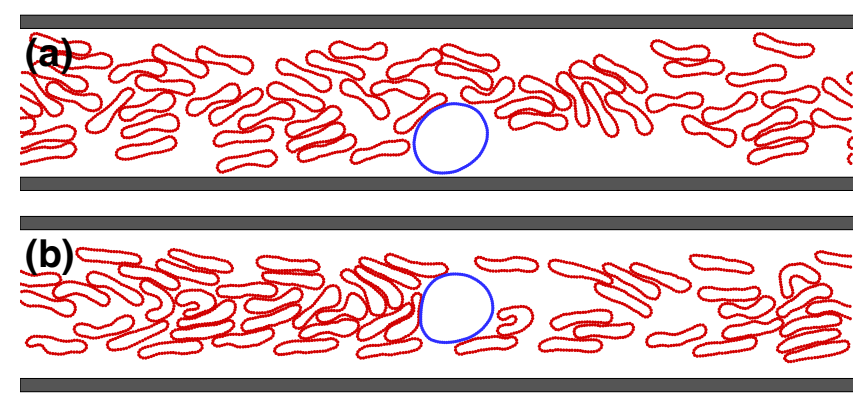

FIG. 1 (color online). Simulation snapshots of the flow (from left to right) at $H_{t}=0.35$ and dimensionless shear rate (a) $\dot{\gamma}^{*}=1.59$ and (b) $\dot{\gamma}^{*}=19.68$. The large quasispherical particle is the WBC [15]. 
Ref. [15]. In particular, we show that there is a pronounced effect of hematocrit on WBC margination and provide new insights into the physical mechanism. We find a reentrant behavior, where margination first increases with increasing hematocrit but then decreases again at higher hematocrits. Furthermore, changes in WBC deformability strongly affect margination, since a deformed WBC may significantly depart from the spherical shape.

Models and methods. - We employ the dissipative particle dynamics method [16], a particle-based mesoscopic simulation technique, to model blood cells suspended in a fluid. A dissipative particle dynamics system consists of $N$ point particles, which interact through pairwise soft forces and move according to Newton's second law of motion. Both two-dimensional cell types are modeled by a collection of $N_{v}$ particles (50 for RBCs and 100 for WBCs) [15] connected by $N_{s}=N_{v}$ springs with the potential [17]

$$
V_{s p}=\sum_{j \in 1 \ldots N_{s}}\left[\frac{k_{B} T l_{m}\left(3 x_{j}^{2}-2 x_{j}^{3}\right)}{4 p\left(1-x_{j}\right)}+\frac{k_{p}}{l_{j}}\right]
$$

where $l_{j}$ is the length of the spring $j, l_{m}$ is the maximum spring extension, $x_{j}=l_{j} / l_{m}, p$ is the persistence length, $k_{B} T$ is the energy unit, and $k_{p}$ is the spring constant. A balance between the two force terms in Eq. (1) determines a nonzero equilibrium spring length $l_{0}$. The cell model also incorporates a bending energy

$$
V_{\text {bend }}=\sum_{j \in 1 \ldots N_{v}} k_{b}\left[1-\cos \left(\theta_{j}\right)\right],
$$

where $k_{b}$ is the bending constant and $\theta_{j}$ is the instantaneous angle between two adjacent springs having the common vertex $j$. In addition, a constraint to maintain a constant cell area is imposed on each cell by the potential $V_{\text {area }}=k_{a}\left(A-A_{0}\right)^{2} / 2$, where $k_{a}$ is the area constraint coefficient, $A$ is the instantaneous cell area, and $A_{0}$ is the specified (target) area. The combination of $A_{0}$ and the cell perimeter $N_{s} l_{0}$ determines the shape of a cell. The RBC diameter is chosen to be $D_{r} \equiv 2 \sqrt{A_{0} / \pi}=0.417 D_{w}$, where $D_{w}$ is the WBC diameter in equilibrium. The RBC equilibrium spring length is $l_{0}=0.0384 D_{w}$ and $l_{m} / l_{0}=2.2$. The RBC shape is biconcave, while WBCs have a circular shape in equilibrium. In all simulations, $k_{b}=50 k_{B} T$ for RBCs and $k_{b}=500 k_{B} T$ for WBCs. The blood cells (72 RBCs and one WBC at $\left.H_{t}=0.45\right)$ are suspended in plasma, which is modeled by a collection of solvent particles.

We consider Poiseuille flow in the channel of width $W=2 D_{w}$, which is driven by a constant force applied to the solvent particles equivalently to a constant pressure gradient. To characterize the flow, we define a dimensionless shear rate as

$$
\dot{\gamma}^{*}=\frac{\eta D_{r}^{3} \overline{\dot{\gamma}}}{\kappa_{r}}=\tau \overline{\dot{\gamma}}
$$

where $\bar{\gamma}=Q / W^{2}$ is the average shear rate, $Q$ is the flow rate, $\eta$ is the solvent viscosity, $\kappa_{r}=k_{b} l_{0}$ is the RBC bending rigidity, and $\tau=\eta D_{r}^{3} / \kappa_{r}$ is a characteristic relaxation time. The other important nondimensional number, which characterizes the ratio of cell elasticity to bending rigidity, is $\alpha=Y D^{2} / \kappa$, where $Y=$ $\left.\left(-\partial^{2} V_{s p} / \partial l^{2}\right)\right|_{l_{0}}$ is the stretching modulus. $\alpha_{r}=1340$ for RBCs, which was roughly estimated to mimic a RBC stretching experiment [18], while $\alpha_{w}$ is assumed to be about 10 times larger. In addition, to make a WBC less deformable, $A_{0, w}$ is set to be $2 \%$ larger than the area of a circle with diameter $D_{w}$, which implies that the springs of WBCs are initially under a small strain. For comparison in physical units in three dimensions (3D), we assume that $D_{w}=10 \mu \mathrm{m}, \quad D_{r}=4.17 \mu \mathrm{m}, \quad W=20 \mu \mathrm{m}$, $\eta=10^{-3} \mathrm{~Pa} \cdot \mathrm{s}, \quad T=37^{\circ} \mathrm{C}, \quad \kappa_{r}=50 k_{B} T, \quad$ and $Y=$ $18.9 \mu \mathrm{N} / \mathrm{m}$.

Results and discussion.-Figure 2 presents the WBC center-of-mass distributions for various $H_{t}$ values. The flow rate is approximately constant for different $H_{t}$ with $\dot{\gamma}^{*} \approx 3.34$. The corresponding WBC distributions are averaged over the two halves of the channel and indicate WBC margination towards the wall $(y=0)$ with a peak around $y / W=0.25$. The strongest margination effect is observed for a range of $H_{t}=0.25-0.35$ (see also Fig. 1 and Ref. [15]), while WBC margination is attenuated at both lower and higher $H_{t}$ values. As expected at low $H_{t}$, WBC margination is weak due to a low concentration of RBCs. However, at high $H_{t}$ values, the simulated results seem to contradict the main hypothesis for WBC margination: a higher $\mathrm{RBC}$ concentration is expected to lead to more $\mathrm{RBC}$ crowding in the channel center, and therefore to stronger WBC migration towards the wall. Also, we notice that the peak position is slightly closer to the wall for higher $H_{t}$ values; this shift is due to WBC deformability, such that the force on a WBC from flowing RBCs is stronger at a higher $H_{t}$, resulting in a more compressed WBC shape.

To identify the physical mechanism responsible for this observation, we examined the $\mathrm{RBC}$ distribution around a

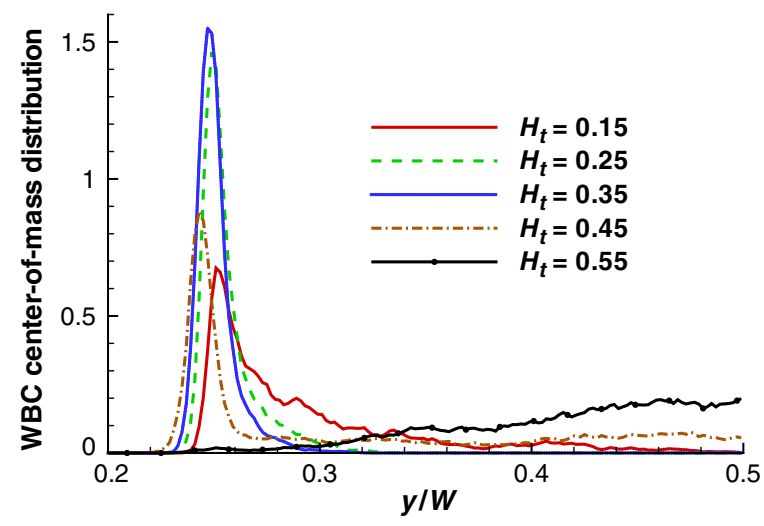

FIG. 2 (color online). WBC center-of-mass distribution for various $H_{t}$ values at $\dot{\gamma}^{*}=3.34$. 


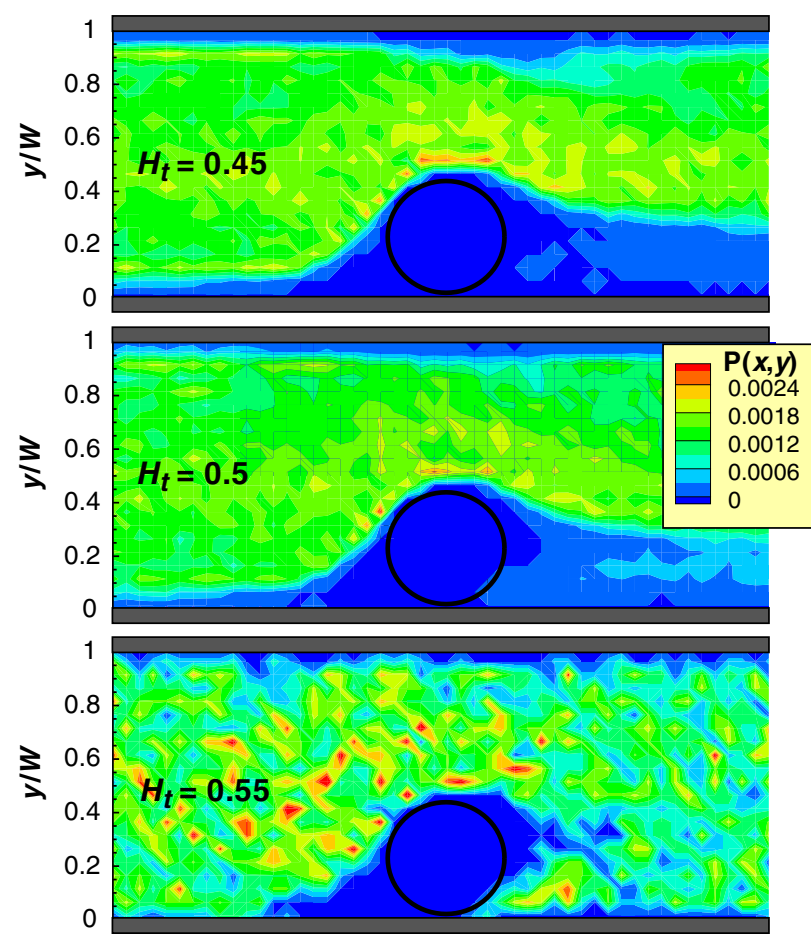

FIG. 3 (color online). RBC center-of-mass distributions around a marginated hard WBC for various $H_{t}$ values at $\dot{\gamma}^{*}=$ 3.34. The circle schematically shows the WBC location. The flow direction is from left to right.

marginated WBC. Figure 3 shows RBC center-of-mass distributions for various $H_{t}$ and $\dot{\gamma}^{*}=3.34$, computed in a comoving coordinate system of the WBC center of mass and with the condition that the WBC center is less than $0.55 D_{w}$ away from one of the walls. This condition is equivalent to the WBC nearly touching the wall. At $H_{t}=$ 0.45 , the region downstream of the WBC near the wall is virtually void of RBCs and it remains free of RBCs during most of the simulated time of about $845 \tau$. As hematocrit is increased to $H_{t}=0.55$, RBCs are found to often enter the region downstream of the WBC due to high RBC crowding. $\mathrm{RBCs}$ in that region move slower than the $\mathrm{WBC}$ next to the wall, so that a RBC may enter the gap between the $\mathrm{WBC}$ and the wall and force the WBC to move away from the wall. This "lift-off" mechanism for the WBC is different from the lift force [19] due to cell-wall hydrodynamic interactions and is governed by the particulate nature of blood. The RBC distributions in Fig. 3 show that such liftoff events occur frequently at $H_{t}=0.55$ and lead to the attenuation of WBC margination illustrated in Fig. 2 and Ref. [15].

Next, the effects of flow rate and WBC deformability on WBC margination are explored. In addition to the previously described setup referred to as "hard" WBCs, we consider both solid (nondeformable) WBCs and "soft" WBCs. The stretching modulus of a soft WBC is 3.2 lower than that of a hard WBC, and the specified area $A_{0}$ is set exactly to $\pi D_{w}^{2} / 4$. The investigated average shear rates are $\dot{\gamma}^{*} \in\{1.57,3.34,5.41,9.34,18.68\}$. Such a thorough study of WBC margination allows us to construct WBC margination diagrams with respect to flow rate and hematocrit, as shown in Fig. 4. The probability of WBC margination is defined as the probability of the WBC center of mass being not further than $0.55 D_{w}$ away from the channel walls. From these plots, we can see that the strongest WBC margination occurs within a certain range of $H_{t}$ values and flow rates. For example, in the case of solid WBCs, WBC margination occurs with high probability for the ranges of $H_{t}=0.2-0.5$ and $\dot{\gamma}^{*}=1-10$. As the WBC becomes softer, the region of high probability for WBC margination shrinks considerably. Specifically, there is a pronounced dependence of WBC margination on flow rate, since a WBC may substantially deform at high flow rates and thus experience a strong lift force from the wall.

Our simulation results are also consistent with experimental observations that WBC adhesion occurs mainly in venules (not in arterioles) in the organism. The characteristic values of $\dot{\gamma}^{*}$ in venules of a comparable diameter are approximately in the range of $\dot{\gamma}^{*}=1-25$, while, in arterioles, $\dot{\gamma}^{*} \gtrsim 30[20,21]$. This estimation is based on the physiological parameters stated above; for example, $\dot{\gamma}^{*}=$ 10 corresponds to the pseudoshear rate of $30 \mathrm{~s}^{-1}$ and the
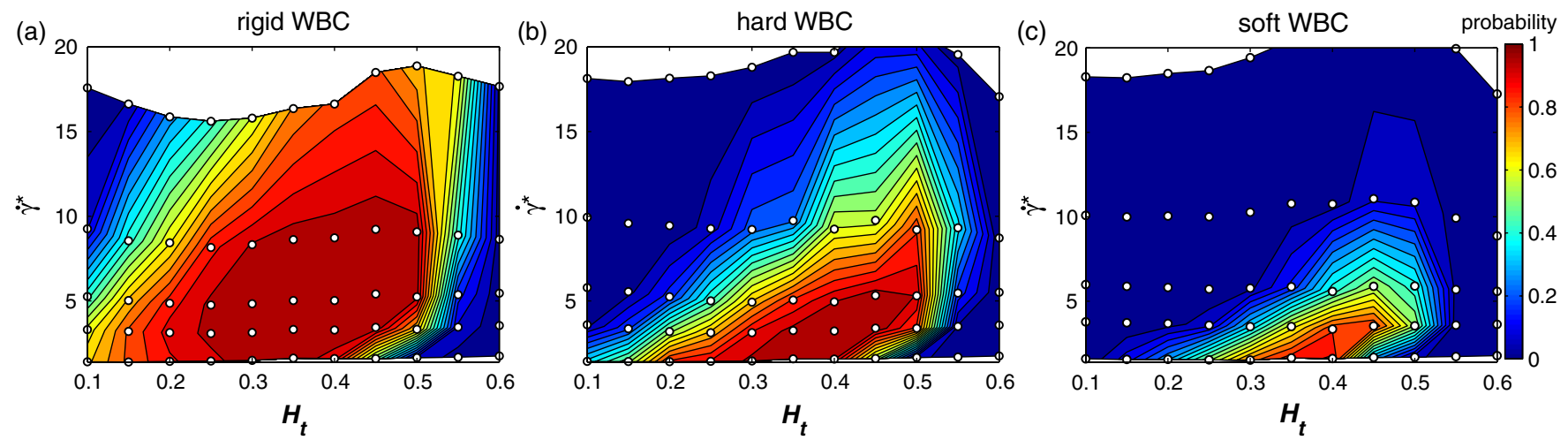

FIG. 4 (color online). Probability diagrams of WBC margination with respect to $\dot{\gamma}^{*}$ and $H_{t}$ for various WBC deformabilities: (a) rigid WBC, (b) hard WBC, and (c) soft WBC. Symbols $(O)$ indicate the values of $H_{t}$ and $\dot{\gamma}^{*}$ for which simulations were performed. 


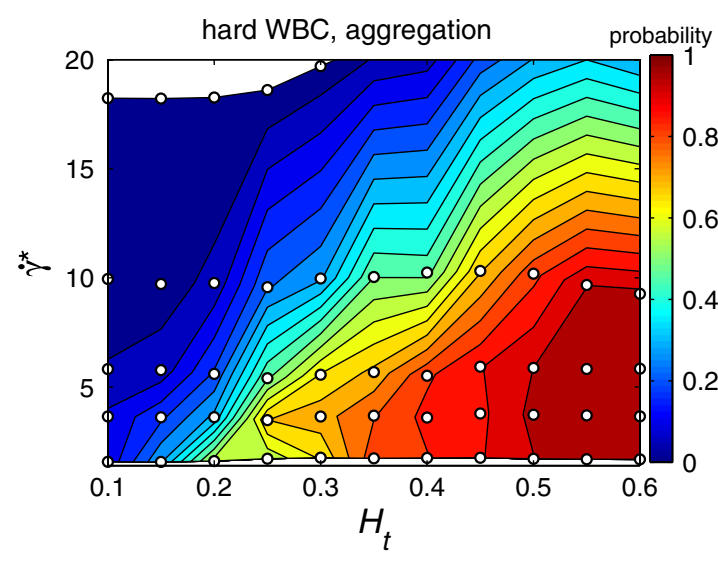

FIG. 5 (color online). Effect of RBC aggregation on WBC margination for the case of hard WBCs. Symbols $(O)$ correspond to the performed simulations.

average flow velocity of $0.6 \mathrm{~mm} / \mathrm{s}$. Thus, efficient WBC margination and consequent adhesion are mainly expected in the venular part of a microvascular network. Our results for the cases of soft and hard WBCs [Figs. 4(b) and 4(c)] show a more narrow range of $\dot{\gamma}^{*}$, where a WBC is marginated in comparison with the range of $\dot{\gamma}^{*}=1-25$. Thus, we conclude that our rigid WBCs in 2D [Fig. 4(a)] provide the most realistic description of WBC margination phenomena in 3D. Of course, predictions of 2D simulations for 3D flows can only be semiquantitative and it is likely that the $\dot{\gamma}^{*}$ range for WBC margination is slightly shifted or expanded in 3D. However, our results are in good agreement with the experiments on leukocyte margination in rectangular microchannels [14], where the optimal hematocrit for WBC margination was identified to be in the range $H_{t}=0.2-0.3$. They are also able to explain discrepancies with previous simulations [13] and experiments [12], which found WBC margination and adhesion to be independent of $H_{t}$, because studied flow rates and $H_{t}$ values in Refs. [12,13] almost entirely fell into the region of strong WBC margination.

$\mathrm{RBC}$ aggregation is believed to enhance WBC margination in blood flow. In our model, aggregation interactions between RBCs are described by a Morse potential, with a strength adjusted to a force in the range of 3-7 pN, as estimated in recent 3D simulations on blood rheology [22]. Figure 5 shows the effect of RBC aggregation on WBC margination for the case of hard WBCs. In comparison with Fig. 4(b), the strong WBC margination region extends now to higher flow rates and $H_{t}$ values. This is likely a consequence of a tighter RBC packing due to the aggregation forces, which result in a less-dispersed $\mathrm{RBC}$ core. The computed $\mathrm{RBC}$ distributions around the $\mathrm{WBC}$ for various $H_{t}$ have confirmed that the region downstream of the WBC remains virtually free of RBCs even for high $H_{t}>0.45$ values due to $\mathrm{RBC}$ aggregation, which substantially reduces the displacing effect of RBCs at high $H_{t}$, as illustrated in Fig. 3. Finally, no significant effect of RBC

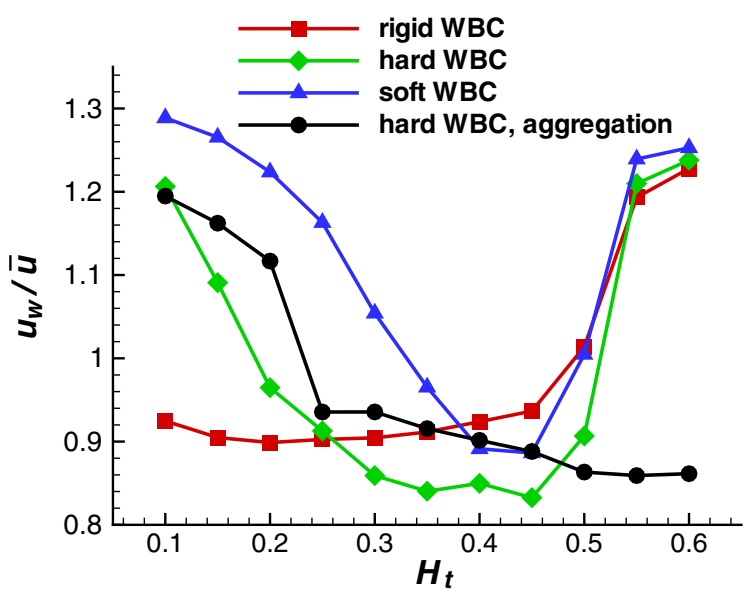

FIG. 6 (color online). WBC average velocity $u_{w}$ for various $H_{t}$ values normalized by the average flow velocity $\bar{u}=Q / W$. The curves for different cases correspond to $\dot{\gamma}^{*} \approx 3.34$.

aggregation on WBC margination is found at high $\dot{\gamma}^{*}$ because RBC aggregates are completely dispersed at high shear rates [22,23].

WBC margination is closely related to WBC flow velocities. Results for the average WBC velocity normalized by the average flow velocity $\bar{u}=Q / W$ as a function of $H_{t}$ are presented in Fig. 6 at $\dot{\gamma}^{*} \approx 3.34$. When margination occurs and the WBC moves near the wall, its velocity is significantly reduced in comparison with a WBC flowing near the center of the channel. In our simulations, the ratio $W / D_{w}=2$ is relatively small, and therefore the velocity difference shown in Fig. 6 is only approximately $30 \%-40 \%$. For larger channel widths, this difference is expected to increase quadratically with $W / D_{w}$. The reduced velocity of marginated WBCs compared to the average blood flow results in a many-fold increase of the WBC concentration in microcirculation (determined by the ratio of the average RBC and WBC velocities). This reduced velocity for marginated WBCs could also play an important role in an efficient adhesion [24,25] because it reduces the required adhesion forces accordingly.

In conclusion, WBC margination strongly depends on flow hydrodynamics (e.g., lift force), as well as on particulate properties of blood, including hematocrit, elasticity characteristics of blood cells, and their interactions. These 2D simulation results will be used to guide realistic 3D simulations in the future, which are able to quantitatively explore the WBC margination phenomena.

D. A.F. acknowledges funding by the Humboldt Foundation.

[1] U. Bagge and R. Karlsson, Microvasc. Res. 20, 92 (1980).

[2] H. L. Goldsmith and S. Spain, Microvasc. Res. 27, 204 (1984).

[3] J.C. Firrell and H.H. Lipowsky, Am. J. Physiol. 256, H1667 (1989). 
[4] K. Ley and T. F. Tedder, J. Immunol. 155, 525 (1995).

[5] R. Alon, D. A. Hammer, and T. A. Springer, Nature (London) 374, 539 (1995).

[6] T. A. Springer, Annu. Rev. Physiol. 57, 827 (1995).

[7] H. L. Goldsmith, G. R. Cokelet, and P. Gaehtgens, Am. J. Physiol. 257, H1005 (1989).

[8] G. R. Cokelet and H. L. Goldsmith, Circ. Res. 68, 1 (1991).

[9] M. Abkarian, C. Lartigue, and A. Viallat, Phys. Rev. Lett. 88, 068103 (2002).

[10] H. L. Goldsmith, Federation Proceedings 30, 1578 (1971).

[11] M. J. Pearson and H. H. Lipowsky, Am. J. Physiol. 279, H1460 (2000).

[12] K. B. Abbitt and G. B. Nash, Am. J. Physiol. 285, H229 (2003).

[13] J. B. Freund, Phys. Fluids 19, 023301 (2007).

[14] A. Jain and L. L. Munn, PLoS ONE 4, e7104 (2009).

[15] See Supplemental Material at http://link.aps.org/ supplemental/10.1103/PhysRevLett.108.028104 for simulation animations of WBC margination.

[16] P. J. Hoogerbrugge and J. M. V. A. Koelman, Europhys. Lett. 19, 155 (1992).
[17] D. A. Fedosov, B. Caswell, and G.E. Karniadakis, Biophys. J. 98, 2215 (2010).

[18] S. Suresh, J. Spatz, J. P. Mills, A. Micoulet, M. Dao, C. T. Lim, M. Beil, and T. Seufferlein, Acta Biomaterialia 1, 15 (2005).

[19] S. Messlinger, B. Schmidt, H. Noguchi, and G. Gompper, Phys. Rev. E 80, 011901 (2009).

[20] A. S. Popel and P. C. Johnson, Annu. Rev. Fluid Mech. 37, 43 (2005).

[21] A. R. Pries, T.W. Secomb, and P. Gaehtgens, Am. J. Physiol. 269, H1713 (1995).

[22] D. A. Fedosov, B. Pan, W. Caswell, G. Gompper, and G. E. Karniadakis, Proc. Natl. Acad. Sci. U.S.A. 108, 11772 (2011).

[23] Q. Zhao, L. G. Durand, L. Allard, and G. Cloutier, Ultrasound Med. Biol. 24, 503 (1998).

[24] M. R. King and D. A. Hammer, Proc. Natl. Acad. Sci. U.S.A. 98, 14919 (2001).

[25] C. Korn and U.S. Schwarz, Phys. Rev. Lett. 97, 138103 (2006). 